\title{
Gilbert Simondon, Imagination et invention (1965-1966)
}

Chatou, Éditions de la Transparence, 2008, 206 pages.

Jean-Hugues Barthélémy

\section{OpenEdition}

Journals

Édition électronique

URL : http://journals.openedition.org/dht/580

DOI : $10.4000 /$ dht.580

ISSN : $1775-4194$

Éditeur :

Centre d'histoire des techniques et de l'environnement du Cnam (CDHTE-Cnam), Société des élèves du CDHTE-Cnam

\section{Édition imprimée}

Date de publication : 31 mars 2009

Pagination : 230-231

ISBN : 978-2-95-30779-3-3

ISSN : 0417-8726

Référence électronique

Jean-Hugues Barthélémy, « Gilbert Simondon, Imagination et invention (1965-1966) », Documents pour I'histoire des techniques [En ligne], 17 | $7^{\text {er }}$ semestre 2009, mis en ligne le 27 septembre 2010, consulté le 22 septembre 2020. URL : http://journals.openedition.org/dht/580 ; DOI : https://doi.org/10.4000/ dht.580

Ce document a été généré automatiquement le 22 septembre 2020.

(C) Tous droits réservés 


\section{Gilbert Simondon, Imagination et invention (1965-1966)}

Chatou, Éditions de la Transparence, 2008, 206 pages.

Jean-Hugues Barthélémy

\section{RÉFÉRENCE}

Gilbert Simondon, Imagination et invention (1965-1966), Chatou, Éditions de la Transparence, 2008, 206 pages.

1 Un an après avoir fait cours sur la perception ${ }^{1}$, Gilbert Simondon livrait un cours intitulé Imagination et invention. Récemment paru pour lui-même et dans son intégralité après avoir fait l'objet de publications partielles et en contexte, ce cours est sans doute la plus importante des publications posthumes de Simondon. Il s'y agit en effet pour ce dernier de soutenir et développer une thèse en vue d'une réforme théorique dont l'ambition peut être cette fois comparée à celles de L'individuation à la lumière des notions de forme et d'information et de Du mode d'existence des objets techniques. Dans ce nouveau cours, les références aux travaux des psychologues sont presque toujours inscrites à l'intérieur de cette visée théorique qui les dépasse, et qui se présente comme fil directeur pour la construction d'une " psychologie "générale" » (p. 138).

2 La continuité entre les deux cours est évidente, et le second renvoie d'ailleurs au premier à l'occasion du traitement des « images intra-perceptives »- plus précisément celles présentes dans la « perception visuelle des formes» (p. 82). Mais cela ne doit pas cacher le fait que le cours Imagination et invention constitue un apport au cours précédent sur le thème même de la perception, puisque ce qui précède la perception, à savoir la motricité du vivant, y est désormais pensé comme naissance d'un «cycle de l'image » qui se prolonge dans la perception elle-même sous la forme des «images intra-perceptives ", puis au-delà de la perception à travers les «images-souvenirs " appelées à devenir "symboles ", pour finalement "concrétiser " l'imagination en invention fondant un «nouveau cycle de rapport avec le réel»(p. 138). Telle est 
justement la thèse nouvelle, par laquelle l'imagination ne sera pas seulement revisitée et élargie par Simondon au nom de l'image, mais se verra également articulée à la thématique de l'invention abordée par Simondon dans Du mode d'existence des objets techniques et développée dans L'invention dans les techniques. De sorte que le cours Imagination et invention définit un carrefour à thématique psychologique au sein du corpus à la fois épistémo-ontologique et technologique de Simondon - comme la Critique de la faculté de juger, dans l'organisation des problématiques philosophiques propres à Emmanuel Kant, définissait le carrefour des Critique de la raison pure et Critique de la raison pratique.

Contrairement au Cours sur la perception, celui sur l'imagination et l'invention est précédé d'un préambule et d'une introduction qui visent à en donner à l'avance la trame théorique, en même temps qu'ils lui apportent une réflexivité et un positionnement - certes allusif - au sein des débats philosophiques et non plus seulement psychologiques sur l'imagination. Mais cette trame et cette réflexivité peuvent parfois être en tension avec le sens des réflexions auxquelles elles introduisent, comme c'est le cas, par exemple, lorsque Simondon écrit en début de Préambule que la " psychologie des facultés » a trop séparé l'imagination, la perception et la mémoire en fonction des "tâches dominantes : anticiper, percevoir, se rappeler " (p. 4). En fait, la séparation des facultés n'est pas une conséquence directe et inéluctable de leur identification à ces "tâches dominantes": Simondon lui aussi thématisera l'imagination comme anticipation, et c'est même l'une des objections qu'il adressera implicitement à Jean-Paul Sartre, qui en effet détachait l'imagination de sa fonction de réalisation par anticipation, c'est-à-dire pour Simondon d'invention au sens strict et positif de ce terme, qui désigne l'ajout d'une réalité objective: "L'imagination comme anticipation n'est plus ainsi une fonction qui détache de la réalité et se déploie dans l'irréel ou le fictif : elle amorce une activité effective de réalisation [...]. La modalité de l'imaginaire est celle du potentiel; elle ne devient celle de l'irréel que si l'individu est privé de l'accès aux conditions de réalisation» (p. 56). La vraie nouveauté sera donc plutôt que Simondon fera de cette anticipation, d'une part ce qui précède et nourrit la perception elle-même - contre toute séparation des facultés -, d'autre part ce qui prolonge les souvenirs-symboles en inventions -en tant qu'ils sont eux aussi des images mais « a posteriori ».

4 Le caractère allusif du positionnement philosophique initial du Cours empêche Simondon d'expliciter dans le détail en quoi sa théorie de l'image est le contrepied parfait de celle de Sartre sur l'imagination - dominante à l'époque en philosophie mais tout juste évoquée - en même temps qu'une subversion de l'opposition entre Sartre et la tradition à laquelle ce dernier s'en prenait. On peut donc rappeler ici que Sartre reprochait à cette tradition de faire de la différence entre perception et imagination une simple différence de degré plutôt que de nature, la perception et l'imagination étant pour Sartre deux modalités de la conscience exclusives l'une de l'autre si l'imagination est une fonction « irréalisante » tandis que la perception est donation de présence. Contre quoi Simondon va proposer ce qui constitue en fait une troisième voie, bien qu'explicitement anti-sartrienne : dans le vivant l'image prend forme dès la motricité et possède donc une modalité pré-perceptive puis intra-perceptive, c'est-àdire a fortiori non-consciente avant d'être consciente - chez Sartre il n'était à chaque fois question que du « sujet conscient ». Où l'on retrouve le souci non-anthropologique de la pensée simondonienne, qui dans L'individuation à la lumière des notions de forme et d'information définissait l'anthropologie comme l'étude essentialiste de l'homme coupé du vivant, et qui à plusieurs reprises dans Imagination et invention pensera l'homme lui- 
même comme susceptible de "régresser » à une "phase » purement instinctive du comportement (pp. 66 et 70).

\section{NOTES}

1. Gilbert Simondon, Cours sur la perception. 1964-1965, préface de Renaud Barbaras, Chatou, Editions de la Transparence, 2006. 